\author{
Yavor Kornovski1, ${ }^{1,}$, Stanislav Slavchev ${ }^{1,2}$, Stoyan Kostov ${ }^{2}$, Yonka Ivanova ${ }^{1,2}$, Angel Yordanov ${ }^{3}$ \\ ${ }^{1}$ Medical University, Varna, Bulgaria \\ ${ }^{2}$ Obstetrics and Gynaecology Clinic, St. Anna University Hospital, Varna, Bulgaria \\ ${ }^{3}$ Department of Gynaecological Oncology, Medical University Pleven, Pleven, Bulgaria
}

\title{
Precancerous lesions of the cervix - aetiology, classification, diagnosis, prevention
}

Address for correspondence:

Associate professor Angel Danchev Yordanov Department of Gynaecologic Oncology,

Medical University Pleven, Bulgaria

e-mail: angel.jordanov@gmail.com

tel.: +359-98-8767-1520

Oncology in Clinical Practice

DOI: $10.5603 /$ OCP.2021.0027

Copyright () 2021 Via Medica

ISSN 2450-1654

e-ISSN 2450-6478

\begin{abstract}
The present review introduces the aetiology and classification of cervical precancers. The principles of diagnosis based on colposcopy are reviewed. The indications for colposcopy and targeted biopsy are steps in the diagnostic process of cervical precancers. Prophylaxis of these diseases prevents cervical cancer as high-grade precancerous lesions represent a direct precursor to cervical cancer. The basics of primary and secondary prevention, the types of screening, and the behaviour of the already-alerted patients after different screenings are presented.

Key words: colposcopy, targeted biopsy, cervical precancerous lesions, cytosmear, HPV vaccines, HPV screening
\end{abstract}

Oncol Clin Pract 2021; 17, 6: 271-276

\section{Characteristics and classification of cervical precancers}

Cervical cancer (CC) has precursors - cell changes can be detected by the so-called screening methods [1,2].

These cellular changes are called dysplasia or CIN - cervical intraepithelial neoplasia (Europe), and SIL - squamous intraepithelial lesion (Bethesda, USA). They are classified as:

- mild dysplasia: CIN1/LGSIL (low-grade SIL);

- severe dysplasia: CIN2, CIN3 / HGSIL (high grade SIL) $[1,2]$.

\section{Aetiology}

Human papillomavirus (HPV) role in cervical carcinogenesis

HPV is one of the main aetiological agents for developing cervical precancers and cervical cancer (CC).
Low-risk and high-risk HPV strains lead to low-grade dysplasia (CIN1/LGSIL1). Only high-risk HPV strains are responsible for disease progression [3].

There are over 100 different HPV subtypes. Only high-risk strains are responsible for cervical carcinogenesis (HPV 16, 18, 26, 31, 33, 35, 39, 45, 51, 52, 56, 58, $59,66)$ and belong to class I carcinogens. HPV 16 and 18 are the two main subtypes associated with cervical cancer. The other important strains vary regionally. HPV 16 contributes to $50-55 \%$ of the cases of invasive cervical cancer. Collectively, HPV 16 and 18 are responsible for approx. $70 \%$ of cervical cancer. This infection is associated with certain risk factors $[4,5]$.

Risk factors for HPV infection:

- sexual behaviour (promiscuity, low sexual culture);

— smoking;

- eating habits;

- immunosuppression.

The so-called asymptomatic HPV carriers happen in 5-20\% of sexually active women of reproductive age. HPV infection is very often reversible. About $90 \%$

\section{Received: 24.04 .2021 \\ Accepted: 11.06 .2021 \\ Early publication date: 08.10 .2021}

This article is available in open access under Creative Common Attribution-Non-Commercial-No Derivatives 4.0 International (CC BY-NC-ND 4.0) license, allowing to download articles and share them with others as long as they credit the authors and the publisher, but without permission to change them in any way or use them commercially. 
of HPV infections can regress spontaneously within 24-36 months [3, 5].

The incidence of HPV infection is $7 \%$ for the age range 20-25 years and less than $2 \%$ for women over 30 years of age. Persistent infection with high-risk HPV strains (16, 18) progresses to HGSIL and cervical cancer. It has been established that HPV infection expresses 2 oncogenes (oncoproteins) - E6 and E7, which in turn inhibit tumour suppressor genes (p53, retinoblastoma rb), causing uncontrolled cell division. Specific indistinct co-factors act as triggers since not every persistent infection with high-risk papillomavirus strains leads to cancer. The transformational period is inconsistent in different patients [3-5].

\section{Diagnosis of cervical precancers}

Histological diagnosis of these lesions is performed in two ways:

1. Colposcopy and targeted biopsy (pinch biopsy under colposcopic control);

2. See-and-treat strategy: in case of inconsistency between cytology (cytologically-signalled patients) and negative/unsatisfactory colposcopic evaluation, LLETZ (large loop excision of the transformation zone) is required, i.e., loop excision providing histological material for diagnosis [6, 7].

Colposcopy plays a key role in the diagnosis and treatment of cervical precancers [8-13].

- Colposcopy allows for the identification, location and outlining of CIN lesions on the cervix, vagina and vulva [8].

- Colposcopy is mandatory for diagnosing and treatment of CIN. It manifests the most susceptive areas where a targeted pinch biopsy should be executed [9]. The main indications for colposcopy are [10]:

1. Abnormal cytology;

2. HPV screening-signalled patients;

3. Contact bleeding. The goals of colposcopy are $[12,13]$ :

1. To identify the site of pinch biopsy that is mostly suspected for HGSIL.

2. To establish the condition of the transformation zone (TZ) and the squamous-cylindrical epithelium border. Assessment of (un)satisfactory colposcopy.

3. To exclude the presence of invasive cervical cancer. Targeted biopsy should always be performed under colposcopic control. Indications for targeted pinch biopsy are:

- before performing ablation based on colposcopic CIN data [14];

- in case of inconsistency between cytology and colposcopic finding [15];

- for histological verification of atypical colposcopic finding (low-grade - Grade 1 and high-grade — Grade 2) (Fig. 1, 2) [14-17].

\section{TZ condition}

A satisfactory (adequate) colposcopic examination is one in which the squamous-cylindrical epithelium border and the borderlines of the atypical epithelium are clearly visible. Unsatisfactory (inadequate) colposcopy is the one with an unclear squamous-cylindrical epithelium border and/or an unclear distal border of an atypical lesion [8-13] (Fig. 3, 4).

\section{Colposcopic signs for early invasion [10]}

1. Size of the lesion: the larger the lesion, especially if it covers the vaginal fornix, the more suspicious of microinvasion it is.

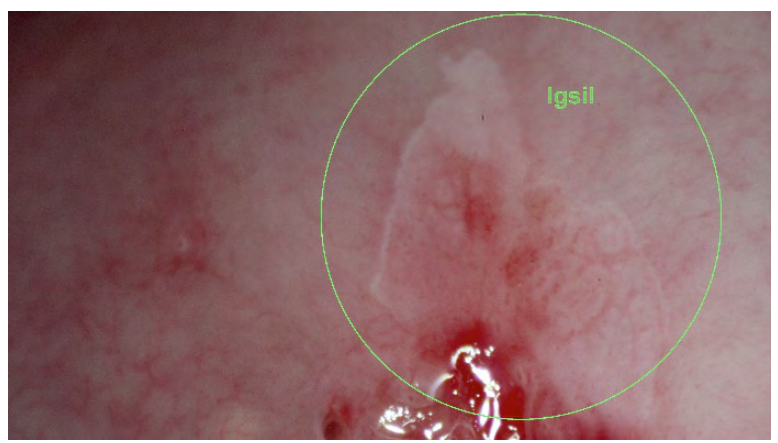

Figure 1. Low-grade Grade1 finding

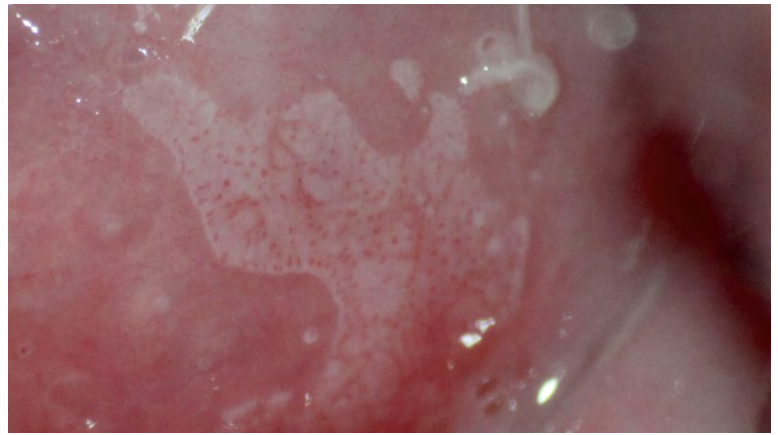

Figure 2. High-grade Grade2 finding

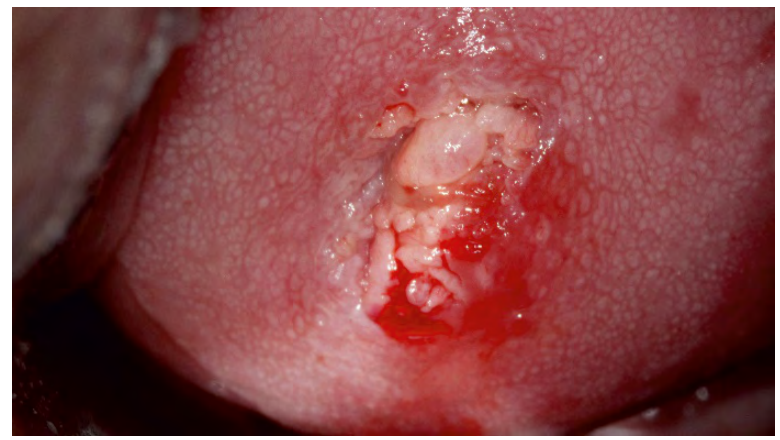

Figure 3. Low-grade atypia with visible borders of the lesion 


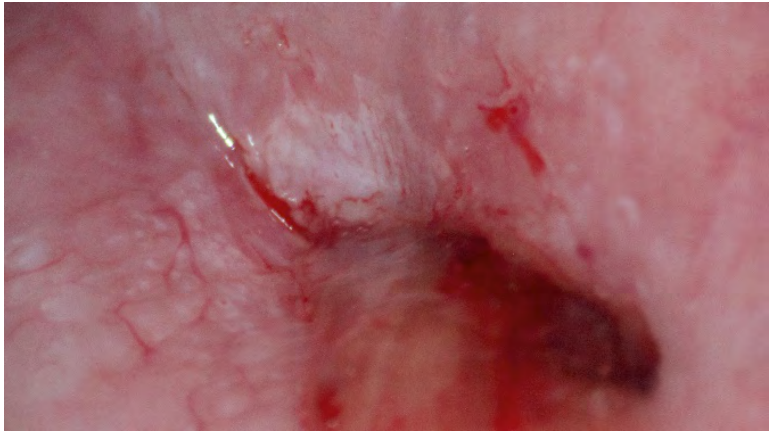

Figure 4. Unclear distal border of the atypical area

2. Different epithelial atypia in one lesion - "lesion in the lesion".

3 . Increased vascularity.

4. Ulceration.

5. Raised edges - "mountain range".

6. Vascular atypia - different in calibre, direction, size.

7. Intense whitening ("chalk whitening") after acetic acid.

\section{Prevention of cervical precancerous lesions (cervical cancer prophylaxis). Primary and secondary prevention}

\section{Primary prevention of cervical cancer}

Cervical cancer has a clear etiological factor: HPV high-risk oncogenic strains. Exposure prevention to this factor is called primary prophylaxis achieved with vaccines against specific human papillomavirus strain. In Bulgaria, there are currently two vaccines available: Silgard - effective against strains $6,11,16,18$, and Cervarix - against strains 16, 18. By 2014, there have been 47 million vaccinated girls, according to WHO and the Global Advisory Committee on Vaccine Safety (GACVS). The safety of 175 million doses has been confirmed till 2013. As of 2014, GACVS has issued no comments on the vaccine's safety. No increased risk of autoimmune diseases, including multiple sclerosis, has been monitored as of 2015/16. More than 120 countries have approved HPV vaccines as part of their immunization calendars. The only recommendation is 15 minutes of follow-up after vaccination due to possible syncope after injection $[18,19]$.

\section{Secondary prevention of cervical cancer}

Detection of the so-called cervical cancer precursors - high-grade lesions CIN (2-3)/HGSIL and preventing their progression to invasive cancer through screening, behaviour signalling, follow-up, and possible treatment. Screening methods (screening programs) for secondary prevention have been developed [20, 21]. There are two types of cervical screening: organized (population-wide), which targets certain groups (by age and frequency/interval of studies), and opportunistic, which is not comprehensive and does not meet the criteria for a screening program. It is applied during a visit to a gynaecologist $[20,21]$.

Criteria for organized screening [20, 21]:

1. Mass screening: includes a specific target population during a specific screening interval.

2. Quality follow-up care and treatment of screening-positive women.

3. Effective communication between the individual components of the screening program (from screening to diagnosis and treatment).

4. High-quality screening tests, diagnostic assessment, treatment and follow-up care.

5. Adequate infrastructure, trained medical staff.

6. Financial resources.

\section{Types of cervical screening}

- HPV high-risk strains screening.

- Cytological - conventional and LBC (liquid-based cytology) [20].

\section{HPV screening}

It is characterized by high sensitivity: negative predictive value (NPV) - sensitivity to CIN3 $>95 \%$, and low specificity - positive predictive value (PPV) [22]. The specificity (PPV) of HPV is lower than that of cytology (Cuzack 2006, Tab. 1).

Randomized trials have reached the following conclusions regarding HPV screening [22, 23]:

- HPV screening is a more productive and cost-effective method for reducing the incidence of cervical cancer.

- HPV screening allows for extended screening intervals but requires a high level of organization.

- HPV-positive patients undergo cytological screening.

- HPV screening is only suitable for women over 30 years of age.

- The integration of organized HPV screening and vaccination will make cervical cancer a rare disease. The European guidelines for quality cervical cancer screening provide the following recommendations for primary HPV screening [22-24]:

Table 1. Sensitivity and specificity according to the cervical screening method

\begin{tabular}{lcc}
\hline Method & Sensitivity [\%] & Specificity [\%] \\
\hline Cytology & 53.0 & 96.3 \\
\hline HPV typing & 96.1 & 90.7 \\
\hline
\end{tabular}


1. HPV screening (with oncogenic HPV tests) can be applied as organized (population) screening

2. The so-called Co-testing (HPV + cytology) should be avoided.

3. Only one primary screening method (HPV or cytology) should be used for the relevant age groups.

4. Routine primary HPV screening may begin over 35 years of age and not earlier than 30 years.

5. There is insufficient evidence of the applicability of this screening for the age group of 30-35 years.

6. HPV screening stops at 60-65 years, and the cytological screening provided that the last result is negative.

7. Cytological screening is used beyond the age range of primary HPV screening.

8. The screening interval after the HPV (-) test is between 5 and 10 years.

\section{Behaviour and follow-up of HPV-alerted patients}

There are three risk groups [25]:

A. Patients positive for HPV 16, 33 strains.

The assessment is for very high risk; colposcopy is required, and readiness for treatment of CIN.

B. Patients positive for HPV 31, 18, 52, 35, 58 strains. The assessment is for high risk and requires colposcopy; HPV 18 causes endocervical lesions.

C. Patients positive for HPV 51, 68, 45, 39, 66, 56, 59 types.

The assessment is for intermediate-risk, and a new

HPV test after one year is recommended.

In some countries, this type of screening is used for the first time [26]. In the United States, HPV positives for HPV16, 18 are referred for colposcopy. Positive for other high-risk HPV strains are subject to cytological examination. Colposcopy is recommended in cytologically-signalled patients. If the smear test is negative, new cytology is performed after one year. HPV-negative patients are subject to a new test in five years. In Belgium and the Netherlands, HPV negatives are subject to a new test in five years. The positives are smeared: if it is smear-positive, patients are referred for colposcopy; if the cytology finding is normal, the test is repeated after one year. If it is still normal, the woman is subject to HPV screening after five years [26]. Similar to the above is the screening system in Italy, where instead of repeated cytology, an HPV test is performed.

\section{HPV screening challenges}

The HPV test is characterized by high sensitivity (NPV) but low specificity. Increasing specificity can be achieved by testing for HPV types $(16,18,33)$ and improving cytology by dual-stain cytology testing for p16 and Ki 67 - this study is a predictor of CIN 2-3. Testing for oncoproteins E6, E7 also complements the screening and manifests that persistent viral infection leads to changes in the cellular regulatory cycle [27, 28].

The combination of vaccination and HPV screening is fundamental to cervical cancer eradication programs [29,30].

HPV vaccination programs include girls aged 12 to 14 years and provide $100 \%$ effectiveness against vaccine strains (if vaccinated before infection). In some countries, boys are also vaccinated [29].

Post-vaccination screening provides for an extended screening interval. In the future, patients will be able to take a screening sample themselves, the so-called self-sampling [29, 30]

\section{Cervical cytology classifications}

\section{Cytological screening systems}

There are three cytological screening systems used in practice [31]:

1. Papanicolaou system or PAP smear test.

2. The Bethesda system (TBS) - 2001, 2014.

3. The British Society for Clinical Cytology (BSCC) classification: used in the UK. According to this system, cellular changes are defined as:

- borderline nuclear changes - BNC (HPV atypia — koilocytosis);

- mild changes - corresponds to CIN1;

- moderate changes - corresponds to CIN2;

- severe changes - corresponds to CIN3.

The Bethesda system grades cellular changes as follows (2014):

1. Non-neoplastic cells.

2. Epithelial cells abnormality - these are cytologically-signalled patients. The changes can affect squamous cells and are described as atypical squamous cells of undetermined significance (ASCUS); atypical squamous cells cannot exclude high-grade squamous intraepithelial lesion (ASC-H); LGSIL, HGSIL; and glandular cells.

\section{Abnormal Cytosmear Recommendations}

Abnormal Cytosmear Recommendations of the American Society for Colposcopy and Cervical Pathology (ASCCP) [32]:

In cases of ASCUS (atypical squamous cells of undetermined significance) or BNA (borderline nuclear abnormality), three lines of behaviour are recommended:

1. Repeat the smear after six months

— if negative, repeat after six months;

- in case of two negative results, return to routine observation;

- if positive (ASCUS, borderline), the patient is referred for colposcopy. 
2. Immediate colposcopy

— in the case of normal colposcopy results - cytology after one year.

3. HPV (DNA) triage:

— HPV positive results - colposcopy;

— HPV negative results — repeated cytology after one year.

In the case of ASC-H (atypical squamous cells - can not exclude high-grade squamous intraepithelial lesion), the patient is referred for colposcopy:

- if the colposcopy result is negative - a new smear test is recommended;

- if the colposcopy and the new cytology are negative cytology after six months is recommended.

In the case of LGSIL/CIN1 (mild dyskaryosis), colposcopy is recommended.

- If the colposcopy result is normal - a new cytology test and/or HPV test is recommended.

- In the case of HGSIL result, the patient is referred for colposcopy and biopsy.

- If the colposcopy is satisfactory and the biopsy does not detect HGSIL, revision of cytology and histology is recommended. If HGSIL is detected - treatment with LLETZ is recommended.

- If the colposcopy is unsatisfactory, it is recommended to perform LLETZ (see-and-treat protocol).

\section{Summary}

Concerning terminology, CIN1 is classified as a low-grade squamous intraepithelial lesion (LGSIL) and CIN2/3 as a high-grade squamous intraepithelial lesion (HGSIL). High-risk HPVs $(16,18,33)$ are responsible for the progression of the carcinogenesis process. Primary prophylaxis (vaccination) is highly effective. Secondary prophylaxis is based on the application of screening systems (HPV high-risk strains and cytology). In screening-positive women, colposcopy is most often performed with or without a biopsy. The diagnosis of precancerous lesions is histological after colposcopic evaluation (targeted biopsy or see-and-treat protocol).

\section{Conflict of interest}

The authors declare no conflict of interest.

\section{References}

1. Ibeanu OA. Molecular pathogenesis of cervical cancer. Cancer Biol Ther. 2011; 11(3): 295-306, doi: 10.4161/cbt.11.3.14686, indexed in Pubmed: 21239888

2. Asiaf A, Ahmad ST, Mohammad SO, et al. Review of the current knowledge on the epidemiology, pathogenesis, and prevention of human papillomavirus infection. Eur J Cancer Prev. 2014; 23(3): 206-224, doi: 10.1097/CEJ.0b013e328364f273, indexed in Pubmed: 24129107.
3. Castle PE, Aslam S, Behrens C. Cervical Precancer and Cancer Risk by Human Papillomavirus Status and Cytologic Interpretation: Implications for Risk-Based Management. Cancer Epidemiol Biomarkers Prev. 2016; 25(12): 1595-1599, doi: 10.1158/1055-9965.EPI-16-0330, indexed in Pubmed: 27587789.

4. Park KJ, Soslow RA. Current concepts in cervical pathology. Arch Pathol Lab Med. 2009; 133(5): 729-738, doi: 10.5858/133.5.729, indexed in Pubmed: 19415947

5. Cantor SB, Atkinson EN, Cardenas-Turanzas M, et al. Natural history of cervical intraepithelial neoplasia: a meta-analysis. Acta Cytol. 2005; 49(4): 405-415, doi: 10.1159/000326174, indexed in Pubmed: 16124170 .

6. Johnson DB, Rowlands CJ. Diagnosis and treatment of cervical intraepithelial neoplasia in general practice. BMJ. 1989; 299(6707): 1083-1086, doi: 10.1136/bmj.299.6707.1083, indexed in Pubmed: 2511973.

7. Long H, Laack N, Gostout B. Prevention, Diagnosis, and Treatment of Cervical Cancer. Mayo Clinic Proceedings. 2007; 82(12): 1566-1574, doi: 10.1016/s0025-6196(11)61104-x.

8. Zeqiri F, Paçarada M, Kongjeli N, et al. The importance of colposcopy in the prevention of cervical malignancies. Int $\mathrm{J}$ Gynaecol Obstet. 2010; 110(2): 149-150, doi: 10.1016/j.ijgo.2010.03.032, indexed in Pubmed: 20471646.

9. Massad LS. Negative colposcopy reduces precancer risk after low-grade cytology. BJOG. 2015; 122(3): 387, doi: 10.1111/1471-0528.12988, indexed in Pubmed: 25055895.

10. Brown $\mathrm{BH}$, Tidy JA. The diagnostic accuracy of colposcopy - A review of research methodology and impact on the outcomes of quality assurance. Eur J Obstet Gynecol Reprod Biol. 2019; 240: 182-186, doi: 10.1016/j.ejogrb.2019.07.003, indexed in Pubmed: 31302386.

11. Zeqiri F, Paçarada M, Zeqiri V, et al. Colposcopy and Cytodiagnosis in the Prevention of Cervical Malignancies. Nigerian Journal of Medicine. 2010; 19(2), doi: 10.4314/njm.v19i2.56507.

12. Świderska-Kiec J, Czajkowski K, Zaręba-Szczudlik J, et al. Comparison of HPV Testing and Colposcopy in Detecting Cervical Dysplasia in $\mathrm{Pa}$ tients With Cytological Abnormalities. In Vivo. 2020; 34(3): 1307-1315, doi: 10.21873/invivo.11906, indexed in Pubmed: 32354923.

13. Luesley D, Downey G. Value of normal colposcopy after an abnormal cervical smear report. J Low Genit Tract Dis. 2009; 13(1): 33-37, doi: 10.1097/LGT.0b013e3181839645, indexed in Pubmed: 19098604.

14. Nakamura $Y$, Matsumoto $K$, Satoh $T$, et al. Optimizing biopsy procedures during colposcopy for women with abnormal cervical cancer screening results: a multicenter prospective study. Int J Clin Oncol. 2015; 20(3): 579-585, doi: 10.1007/s10147-014-0739-6, indexed in Pubmed: 25145298.

15. Gitsch G, Reinthaller A, Tatra G, et al. Diagnosis of cervical intraepithelial neoplasia and human papillomavirus infection: punch biopsy versus cervical smear. Arch Gynecol Obstet. 1991; 249(4): 179-184, doi: 10.1007/BF02390385, indexed in Pubmed: 1665685.

16. Barker B, Garcia F, Lozevski J, et al. The correlation between colposcopically directed cervical biopsy and loop electrosurgical excision procedure pathology and the effect of time on that agreement. Gynecol Oncol. 2001; 82(1): 22-26, doi: 10.1006/gyno.2001.6245, indexed in Pubmed: 11426957.

17. Heatley MK, Bury JP. The correlation between the grade of dyskaryosis on cervical smear, grade of cervical intraepithelial neoplasia (CIN) on punch biopsy and the final histological diagnosis on cone biopsies of the cervix. Cytopathology. 1998; 9(2): 93-99, doi: 10.1046/j.1365-2303.1998.00094.x, indexed in Pubmed: 9577735

18. Arbyn $M, X u L$, Simoens $C$, et al. Prophylactic vaccination against human papillomaviruses to prevent cervical cancer and its precursors. Cochrane Database Syst Rev. 2018; 5: CD009069, doi: 10.1002/14651858.CD009069.pub3, indexed in Pubmed: 29740819.

19. Arbyn M, Xu L. Efficacy and safety of prophylactic HPV vaccines. A Cochrane review of randomized trials. Expert Rev Vaccines. 2018; 17(12): 1085-1091, doi: 10.1080/14760584.2018.1548282, indexed in Pubmed: 30495978

20. Sawaya GF, Smith-McCune K. Cervical Cancer Screening. Obstet Gynecol. 2016; 127(3): 459-467, doi: 10.1097/AOG.0000000000001136, indexed in Pubmed: 26855089.

21. Brousseau EC, Ahn S, Matteson KA. Cervical Cancer Screening Access, Outcomes, and Prevalence of Dysplasia in Correctional Facilities: A Systematic Review. J Womens Health (Larchmt). 2019; 28(12): 1661-1669, doi: 10.1089/jwh.2018.7440, indexed in Pubmed: 30939063 .

22. Arbyn M, Ronco G, Anttila A, et al. Evidence regarding human papillomavirus testing in secondary prevention of cervical cancer. Vaccine. 2012; 30 Suppl 5: F88-F99, doi: 10.1016/j.vaccine.2012.06.095, indexed in Pubmed: 23199969. 
23. Ronco G, Dillner J, Elfström KM, et al. International HPV screening working group. Efficacy of HPV-based screening for prevention of invasive cervical cancer: follow-up of four European randomised controlled trials. Lancet. 2014; 383(9916): 524-532, doi: 10.1016/S01406736(13)62218-7, indexed in Pubmed: 24192252.

24. Lindroth $Y$, Borgfeldt C, Thorn G, et al. Population-based primary HPV mRNA cervical screening compared with cytology screening. Prev Med. 2019; 124: 61-66, doi: 10.1016/j.ypmed.2019.04.021, indexed in Pubmed: 31047910.

25. Polman NJ, Veldhuijzen NJ, Heideman DAM, et al. Management of HPV-positive women in cervical screening using results from two consecutive screening rounds. Int J Cancer. 2019; 144(9): 2339-2346, doi: 10.1002/ijc.32004, indexed in Pubmed: 30565673.

26. Polman NJ Snijders PJF, Kenter GG, et al. HPV-based cervical screening: Rationale, expectations and future perspectives of the new Dutch screening programme. Prev Med. 2019; 119: 108-117, doi 10.1016/i.ypmed.2018.12.021, indexed in Pubmed: 30594536.

27. Dovnik A, Repše-Fokter A. p16/Ki-67 immunostaining in the triage of young women with LSIL, ASC-US, and ASC-H cytology. Diagn
Cytopathol. 2020; 48(1): 96-97, doi: 10.1002/dc.24339, indexed in Pubmed: 31714671

28. Zhu Y, Ren C, Yang Li, et al. Performance of p16/Ki67 immunostaining, HPV E6/E7 mRNA testing, and HPV DNA assay to detect high-grade cervical dysplasia in women with ASCUS. BMC Cancer. 2019; 19(1): 271, doi: 10.1186/s12885-019-5492-9, indexed in Pubmed: 30917784.

29. Bogani G, Serati M, Leone Roberti Maggiore U, et al. Cervical intraepithelial neoplasia in women who had vaccination against HPV. Int J Gynaecol Obstet. 2019; 147(2): 233-237, doi: 10.1002/ijgo.12934, indexed in Pubmed: 31361908.

30. Canfell K. Towards the global elimination of cervical cancer. Papillomavirus Res. 2019; 8: 100170, doi: 10.1016/j.pvr.2019.100170, indexed in Pubmed: 31176807

31. Nkwabong E, Laure Bessi Badjan I, Sando Z. Pap smear accuracy for the diagnosis of cervical precancerous lesions. Trop Doct. 2019; 49(1): 34-39, doi: 10.1177/0049475518798532, indexed in Pubmed: 30222058.

32. Apgar BS, Brotzman G. Management of cervical cytologic abnormalities. Am Fam Physician. 2004; 70(10): 1905-1916. 